\title{
Infección por Cystoisospora belli en pacientes con VIH: análisis de casos con diferente evolución clínica
}

\author{
Heber Silva-Díaz, Darwin Fernández-Valverde, Gustavo Hernández-Córdova y Virgilio E. Failoc-Rojas
}

\section{Cystoisospora belli infection in HIV patients: Analysis of cases with different clinical evolution}

Introduction: Cystoisospora belli infection is one of the most important causes of watery diarrhea in patients with HIV and causes high rates of morbidity and mortality. Methods: A retrospective study was conducted in patients with HIV and diarrhea by $C$. belli. Clinical and laboratory characteristics were collected by analyzing clinical records. Results: Four had recurrent diarrhea despite receiving secondary prophylaxis with cotrimoxazole and specific treatment, two of which had a good viral and immunological response to highly active antiretroviral therapy (HAART) at the time of diagnosis and antiparasitic treatment. While the remaining three did not receive prophylaxis, neither did HAART (two of them), but they responded well to treatment. Conclusions: C. belli is an important cause of diarrhea in HIV patients on HAART and prophylaxis, being able to have different clinical evolution. We suggest that persistent infection may be due to drug failure by intrinsic or extrinsic to the parasite causes, or to defects in restoration of the intestinal immune system, or both.

Key words: Cystoisosporiasis; isosporiasis; diarrhea; HIV infections; disease eradication; treatment failure.

Palabras clave: Cystoisosporiasis; isosporiasis; diarrea; infecciones por VIH; erradicación de la enfermedad; falla terapéutica.

\section{Introducción}

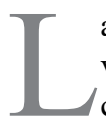

as infecciones oportunistas en pacientes con el virus de la inmunodeficiencia humana (VIH) son causa frecuente de morbi-mortalidad, entre las que destacan las de etiología parasitaria ${ }^{1}$. Cystoisospora belli (syn. Isospora belli), es un protozoo del Subphylum Apicomplexa, Levine,1970. Capítulo del Dr. Jacob K. Frenkel (creador del Género Cystoisospora) del Libro Parasitología Humana de Werner Apt, 2013, de infección intracelular obligado, principalmente de la mucosa intestinal de pacientes inmunocomprometidos. No obstante, en la infección por VIH, es donde $C$. belli ha manifestado su mayor frecuencia e importancia. Al respecto, dos estudios en Perú revelaron que la cystoisosporosis $(8,4$ a $10,6 \%)$ fue una de las infecciones intestinales más frecuentes en este grupo de pacientes ${ }^{2,3}$. La enfermedad se caracteriza por diarrea acuosa y recurrente, dolor abdominal y pérdida ponderal ${ }^{4}$. La diseminación extra-intestinal ha sido poco reportada ${ }^{5,6}$.

La introducción de la terapia anti-retroviral de gran actividad (TARGA) en los últimos años, ha mejorado la capacidad de respuesta inmunológica y disminuido la carga viral. Adicional a ello, el uso extensivo de profilaxis secundaria con cotrimoxazol, ha permitido disminuir las infecciones oportunistas y las tasas de mortalidad asociadas al VIH, principalmente en países en desarrollo ${ }^{7,8}$. Sin embargo, C. belli, continúa siendo frecuente y es uno de los parásitos más importantes en pacientes con infección por VIH en América Latina ${ }^{2,9}$.

En el presente estudio se describen siete casos de diarrea por $C$. belli en pacientes infectados con VIH. Además se discute la relación del fallo al tratamiento y las recurrencias con el TARGA, profilaxis secundaria y aspectos inmunológicos.

\section{Métodos}

Se realizó un estudio retrospectivo en pacientes con infección por VIH y C. belli admitidos en el Hospital Regional Lambayeque (categoría III), Chiclayo, Perú, desde enero de 2013 hasta febrero de 2016. Una vez identificados los casos, se realizó un análisis documental de las historias clínicas registrando características clínicas (edad, sexo, infección concomitante, número de episodios de diarrea, evolución), terapéuticas (TARGA, profilaxis secundaria) y de laboratorio (hemograma, carga viral, linfocitos (LT) CD4+, coloración de Ziehl-Neelsen modificado).

Se consideró caso clínico de infección por $C$. belli si hubo informe de laboratorio con coloración de Ziehl-Neelsen modificado en heces positivo a ooquistes del parásito.

El estudio fue revisado y aprobado por el Comité de Ética en Investigación del Hospital Regional Lambayeque.
Hospital Regional Lambayeque, Chiclayo-Perú. Laboratorio de Parasitología, Metaxénicas y Zoonosis (HSD, DFV).

Universidad Peruana Cayetano Heredia, Lima-Perú (GHC). Universidad Nacional Pedro Ruíz Gallo, Lambayeque, Perú. Facultad de Medicina Humana (VEFR).

Trabajo autofinanciado. Los autores declaran no tener conflicto de interés.

Recibido: 12 de octubre de 2016 Aceptado: 17 de julio de 2017

Correspondencia a: Heber Silva-Díaz hsilva@hrlamb.gob.pe 


\section{Resultados}

Siete pacientes infectados con VIH fueron identificados con infección por $C$. belli, demostrado por la presencia de ooquistes del parásito (Figura 1). Cinco fueron varones, tuvieron una media de edad de 32 años y diarrea crónica. Cuatro habían recibido TARGA y profilaxis secundaria por más de un año. Las características clínicas y de laboratorio de los siete casos se resumen en la Tabla 1. Tres casos son detallados a continuación.

\section{Caso 1}

Varón de 45 años, en TARGA hacía cinco años, tuberculosis desde el 2011 y hepatitis C. Ingresó en agosto de 2013 con diarrea acuosa asociada a vómitos y dolor abdominal y un tiempo de enfermedad de tres semanas, con un peso de $41 \mathrm{~kg}$ y parámetros hematológicos y signos vitales normales. Recibió tratamiento de rehidratación isotónica, junto con cotrimoxazol $2.880 \mathrm{mg}$ / día VO y ciprofloxacina $400 \mathrm{mg}$ /día EV durante seis días, medicamentos usados regularmente contra diarrea en pacientes con infección por VIH. Fue dado de alta por mejoría clínica. Entre el año 2014 y la actualidad, el paciente presentó múltiples recurrencias diarreicas, siendo 10 episodios los más graves. Las dos últimas recurrencias fueron en mayo y octubre del año 2015; se hizo un estudio copro-parasitológico con la coloración de
Ziehl-Neelsen modificado, encontrando $C$. belli en ambas oportunidades, a pesar de tener una buena respuesta al TARGA y recibir profilaxis secundaria con cotrimoxazol y ciprofloxacina para prevenir recidivas por $C$. belli. En la primera oportunidad recibió tratamiento con cotrimoxazol $3.200 \mathrm{mg} /$ día (de sulfa) VO, ciprofloxacina $2.000 \mathrm{mg} /$ día VO y metronidazol $1.500 \mathrm{mg} /$ día; y la segunda vez recibió nitazoxanida $500 \mathrm{mg} /$ día VO, además de continuar con cotrimoxazol y ciprofloxacina a la misma dosis. A la fecha el paciente continúa con recurrencias periódicas.

\section{Caso 2}

Varón de 26 años, con TARGA, profilaxis de cotrimoxazol y en tratamiento para tuberculosis. Ingresó en diciembre de 2014 con diarrea persistente, afebril y con ligera palidez. Su presión arterial fue de 100/60 $\mathrm{mmHg}$, signos vitales normales, peso de $36 \mathrm{~kg}$ y hemograma normal. Refirió que en el último mes y medio había perdido $10 \mathrm{~kg}$ de peso. Recibió metronidazol $1.500 \mathrm{mg} /$ día EV para tratamiento de la diarrea. El estudio coprológico por coloración de Ziehl-Neelsen modificado reveló ooquistes de $C$. belli y se le adicionó cotrimoxazol $2.880 \mathrm{mg} /$ día VO por 10 días, mejorando clínicamente. Dos semanas después, el paciente ingresó por recurrencia de la diarrea y deshidratación grave e hipotensión arterial (70/30 $\mathrm{mmHg}$ ). Fue tratado con ciprofloxacina $2.000 \mathrm{mg} /$ día EV y cotrimoxazol $2.880 \mathrm{mg} /$ día y mejoró después de

Figura 1. Ooquiste inmaduro de C. belli: A. Con esporoblasto (Tinción ácido-resistente modificada); B. Sin esporoblasto (tinción ácido-resistente modificada) y C. Con esporoblasto (examen directo); D. Cristales de Charcot Leyden asociados a la infección por C. belli (examen directo).

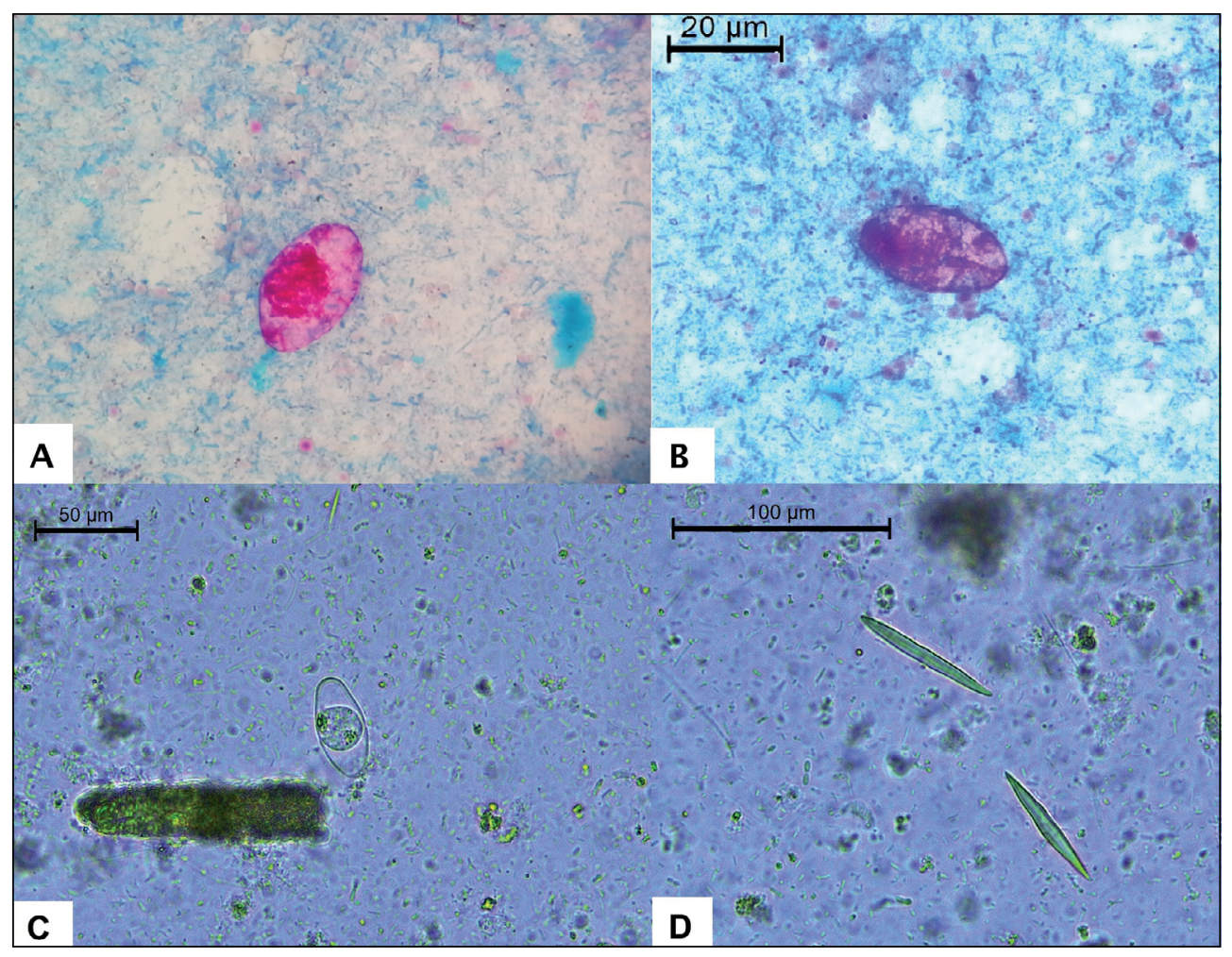




\begin{tabular}{|c|c|c|c|c|c|c|c|}
\hline Número de caso & 1 & 2 & 3 & 4 & 5 & 6 & 7 \\
\hline Sexo/edad (años) & $M / 45$ & $\mathrm{M} / 26$ & $M / 37$ & $M / 35$ & $\mathrm{M} / 19$ & $F / 24$ & $F / 36$ \\
\hline $\begin{array}{l}\text { Diarrea } \\
\text { (n de episodios) }\end{array}$ & Sí (10) & Sí (4) & Sí (3) & Sí (8) & Sí (1) & Sí (1) & Sí (1) \\
\hline $\begin{array}{l}\text { Carga viral } \\
\text { (copias ARN/mL) * }\end{array}$ & 1647 & No detectado & 110986 & 246879 & 282286 & 161185 & 206 \\
\hline $\begin{array}{l}\text { CD4+ } \\
\left(\text { cés } / \mathrm{mm}^{3}\right)^{*}\end{array}$ & 309 & 309 & 227 & 158 & 143 & 2 & 42 \\
\hline $\begin{array}{l}\text { TARGA } \\
\text { (tiempo en meses)* }^{*}\end{array}$ & Sí (60) & Sí (13) & Sí (12) & Sí (81) & $\begin{array}{l}\text { No (recién diag- } \\
\text { nosticado) }\end{array}$ & $\begin{array}{l}\text { No (recién diag- } \\
\text { nosticado) }\end{array}$ & Sí (1) \\
\hline $\begin{array}{l}\text { Tratamientos con } \\
\text { cotrimoxazol } \\
\text { recibidos }\end{array}$ & $\begin{array}{c}\text { Siete: dos (único) } \\
\text { y cinco (asociado } \\
\text { a CIP o } \\
\text { nitazoxanida) }\end{array}$ & Dos (único) & Dos (único) & $\begin{array}{c}\text { Tres: dos (único) } \\
\text { y uno (asociado } \\
\text { a CIP) }\end{array}$ & Uno (único) & Uno (único) & Uno (único) \\
\hline Evolución & $\begin{array}{c}\text { Diarrea persiste } \\
\text { con recurrencias } \\
\text { periódicas }\end{array}$ & $\begin{array}{l}\text { Fallecido por las } \\
\text { complicaciones }\end{array}$ & $\begin{array}{c}\text { Diarrea persiste } \\
\text { con síntomas } \\
\text { leves }\end{array}$ & $\begin{array}{c}\text { Diarrea persiste } \\
\text { con recurrencias } \\
\text { periódicas }\end{array}$ & $\begin{array}{l}\text { Síntomas } \\
\text { resueltos }\end{array}$ & $\begin{array}{l}\text { Síntomas } \\
\text { resueltos }\end{array}$ & $\begin{array}{l}\text { Síntomas } \\
\text { resueltos }\end{array}$ \\
\hline
\end{tabular}

*Características clínicas cuando el parásito fue detectado por primera vez (C. belli). Leu = leucocitos sanguíneos (células/uL); Hb = hemoglobina ( $\mathrm{g} / \mathrm{dL}$ ); Eo = eosinófilos sanguíneos (\%); SXT = cotrimoxazol; CIP = ciprofloxacina; $A B C=$ Abacavir; $3 T C=$ Lamivudina; NVP = Nevirapina; ETV = Etravirina; $\mathrm{EFV}=$ Efavirenz; TDF $=$ Tenofovir; ATV = Atazanavir.

una semana por lo que fue dado de alta con profilaxis de cotrimoxazol $1.600 \mathrm{mg} /$ día (de sulfa) VO para prevenir recaídas. En enero de 2015 volvió a ingresar por sala de emergencias presentando el mismo cuadro, pero falleció a los pocos días, a pesar de su reconstitución inmune y tratamiento a dosis altas de ciprofloxacina, cotrimoxazol, metronidazol; además de ceftazidima y amikacina.

\section{Caso 5}

Varón de 19 años, que ingresó en agosto de 2015 por diarreas acuosas, un peso de $65 \mathrm{~kg}$ y presión arterial de 100/60 mmHg. El examen serológico se demostró infección por VIH y sífilis. El estudio copro-parasitológico detectó quistes de Entamoeba histolytica / E. dispar, quistes de Entamoeba coli, quistes de Endolimax nana $\mathrm{y}$ trofozoitos de Blastocystis hominis, mientras que la coloración de Ziehl-Neelsen modificado demostró ooquistes de $C$. belli y Cryptosporidium sp. Se trató con cotrimoxazol $2.880 \mathrm{mg}$ /día VO y metronidazol 1.500 $\mathrm{mg}$ /día VO por siete días, e inició TARGA y tratamiento contra la sífilis. El paciente resolvió los síntomas y en los controles posteriores mostró un peso normal, refiriendo no presentar molestias intestinales.

\section{Discusión}

En el presente estudio, se presentan siete casos de pacientes con infección por VIH y diarrea por C. belli. Dos de ellos recientemente diagnosticados por lo que no recibían TARGA y un paciente que había iniciado un mes anterior al diagnóstico de C. belli, pero los tres no habían recibido profilaxis secundaria con cotrimoxazol y sus concentraciones de LT CD4+ eran inferiores a 200 céls $/ \mathrm{mm}^{3}$. No obstante, respondieron favorablemente al tratamiento contra $C$. belli, sin presentar recurrencias. Mientras que los otros cuatro pacientes, recibían TARGA, profilaxis secundaria y tres de ellos presentaron evidencia de restauración inmunológica $(>200$ L T CD4+/mm 3 ); sin embargo, el tratamiento estándar falló en la erradicación 
del parásito. Esta contradicción clínica ha sido reportada anteriormente con algunas particularidades ${ }^{10-12}$.

En Perú, un estudio demostró que los coccidios intestinales se han encontrado con mayor frecuencia en pacientes con infección por $\mathrm{VIH}^{3}$. Asimismo, en una serie de casos realizado en Francia en pacientes infectados con VIH y TARGA, $C$. belli se encontró en aquellos con concentraciones de LT CD4+ de 154 céls $/ \mathrm{mm}^{3}$, a diferencia de otros coccidios como Cryptosporidium spp. con 24 LT CD4+/mm ${ }^{3} 12$.

De los pacientes que tenían diarrea recurrente asociado a $C$. belli (casos 1 a 4 de Tabla 1), se observó que tenían buena respuesta inmunológica al TARGA; incluso, el único paciente que falleció tenía carga viral indetectable y LT CD4+ mayor a 300 céls $/ \mathrm{mm}^{3}$, lo que se podría explicar por las siguientes hipótesis:

- Deficiente respuesta defensiva por parte del sistema inmune intestinal. Esto debido al deterioro celular (LT CD4+ y LT Th17) por la infección viral temprana y a su incompleta restauración, incluso con TARGA ${ }^{13}$.

- Desarrollo de mecanismos de resistencia farmacológica. Evento posible debido al entorno de presión selectiva que promueve la profilaxis secundaria ${ }^{7}$. Si bien, se ha demostrado la emergente resistencia de algunos protozoos humanos a fármacos de uso común ${ }^{14}$, es necesario más estudios que muestren mayores evidencias de la resistencia de $C$. belli a fármacos como cotrimoxazol y ciprofloxacina.

- Reclusión del parásito en sitios extra-intestinales en forma de quistes unicelulares, a partir del cual podría re-infectar la mucosa intestinal en determinadas circunstancias $^{5,6}$.

- Reinfección debido a la ingestión de alimentos contaminados con ooquistes esporulados de C. belli, lo que es posible en países en desarrollo y endémicos, como Perú.

- Malabsorción intestinal de fármacos debido al deterioro del sistema digestivo. Sin embargo; también se ha visto fallo al tratamiento parenteral y no se corresponde con la buena respuesta al TARGA administrado vía oral.

- Inadecuada adherencia al tratamiento profiláctico y al TARGA.

Por otro lado, la persistencia de $C$. belli en tres pacientes con más de 200 céls $/ \mathrm{mm}^{3}$ de LT CD4+ observado en este estudio, indican la necesidad de reevaluar las concentraciones convencionales de este parámetro, por cuanto a partir de éste, se toman decisiones clínicas para el manejo del paciente con infección por VIH. Al respecto, un estudio reciente concluye que, la iniciación de TARGA en infección temprana, cuando el paciente presenta más de $500 \mathrm{LT} \mathrm{CD} 4+/ \mathrm{mm}^{3}$, provee beneficios sobre aquellos que lo inician después, en niveles inferiores a $350 \mathrm{LT} \mathrm{CD} 4+/ \mathrm{mm}^{315}$. En este sentido, el inicio temprano del tratamiento anti-retroviral evitaría el agotamiento de LT CD4+ y mantendría el fenotipo Th17 intestinal, permitiendo la restauración inmunológica intestinal y consecuente mejoría clínica ${ }^{15,16}$.

El medicamento de elección para el tratamiento y profilaxis de la infección por $C$. belli es cotrimoxazol forte (160 mg de trimetoprim y $800 \mathrm{mg}$ de sulfametoxazol), pero varía de acuerdo al estado de inmunocompromiso ${ }^{7,17}$. Sin embargo, también se ha descrito el uso de ciprofloxacina y nitazoxanida ${ }^{10,11,17}$. El control de la infección por $C$. belli, así como de otras infecciones oportunistas, requerirá probablemente de una nueva generación de fármacos específicos o el mejor uso de los mismos, así como el manejo integral del paciente. Al respecto, un estudio previo mostró que la competencia inmune y la terapia antimicrobiana específica en conjunto mejoran la respuesta contra los enteropatógenos ${ }^{1}$.

En conclusión, la infección por $C$. belli es causa importante de diarrea en pacientes infectados con VIH, observándose distinta evolución clínica. Asimismo, algunos pacientes podrían experimentar infección persistente y diarrea recurrente a pesar de una adecuada respuesta al TARGA y profilaxis secundaria con cotrimoxazol, tal como también ha sido reportado en otras series de $\operatorname{casos}^{10,11}$. Las causas de la persistencia de la infección son poco conocidas, pero puede estar relacionado al fallo farmacológico por causas intrínsecas o extrínsecas al parásito, o a defectos en la restauración del sistema inmune intestinal.

\section{Resumen}

Introducción: La infección por Cystoisospora belli es una de las causas más frecuentes de diarrea acuosa en pacientes con infección por VIH y ocasiona altas tasas de morbilidad y mortalidad. Métodos: Se realizó un estudio retrospectivo en pacientes infectados con VIH y diarrea por $C$. belli. Características clínicas y de laboratorio fueron recolectadas mediante el análisis de historias clínicas. Resultados: Se identificaron siete casos. Cuatro presentaron diarrea recurrente a pesar de recibir profilaxis secundaria con cotrimoxazol y tratamiento específico, dos de los cuales tuvieron buena respuesta viral e inmunológica al tratamiento anti-retroviral de gran actividad (TARGA) al momento del diagnóstico y tratamiento antiparasitario. Mientras que, los tres restantes no recibían profilaxis, tampoco TARGA (dos de ellos), pero respondieron bien al tratamiento. Conclusiones: $C$. belli es causa importante de diarrea en pacientes VIH con TARGA y profilaxis, pudiendo tener distinta evolución clínica. Sugerimos que la infección persistente puede ser debido al fallo farmacológico por causas intrínsecas o extrínsecas al parásito, o a defectos en la restauración del sistema inmune intestinal, o ambos. 


\section{Referencias bibliográficas}

1.- Jha A K, Uppal B, Chadha S, Aggarwal P, Ghosh R, Dewan R. Enteric pathogens, immune status and therapeutic response in diarrhea in HIV/AIDS adult subjects from north India. Curr HIV Res 2013; 11 (4): 326-32.

2.- García C, Rodríguez E, Do N, López de Castilla D, Terashima A, Gotuzzo E. Parasitosis intestinal en el paciente con infección VIHSIDA. Rev Gastroenterol Perú 2006; 26 (1) 21-4.

3. Omayra Chincha L, Bernabé-Ortiz A, Samalvides F, Soto L, Gotuzzo E, Terashima A. Infecciones parasitarias intestinales y factores asociados a la infección por coccidias en pacientes adultos de un hospital público de Lima, Perú. Rev Chilena Infectol 2009; 26 (5): 440-4.

4.- Dillingham R, Houpt E R. Cystoisospora belli (syn. Isospora belli). Hunter's Tropical Medicine and Emerging Infectious Disease. Elsevier; 2013. p. 683-4.

5.- Takahashi H, Falk G A, Cruise M, Morris-Stiff G. Chronic cholecystitis with Cystoisospora belli in an immunocompetent patient. Br Med J Case Rep. 2015; 2015.

6.- Frenkel J K, Silva M B de O, Saldanha J C, de Silva-Vergara M L, Correia D, Barata C H, et al. Extraintestinal finding of Isospora belli unizoic cysts in a patient with AIDS: case report. Rev Soc Bras Med Trop 2003; 36 (3): 409-12.

7.- $\quad$ Cheng W, Wu Y, Wen Y, Ma Y, Zhao D, Dou Z, et al. Cotrimoxazole prophylaxis and antiretroviral therapy: an observational cohort study in China. Bull World Health Organ 2015; 93 (3): 152-60

8.- $\quad$ Church J A, Fitzgerald F, Walker A S, Gibb D M, Prendergast A J. The expanding role of co-trimoxazole in developing countries. Lancet Infect Di. 2015; 15 (3): 327-39.

9.- Cardoso L V, Galisteu K J, Júnior A S, Chahla L A, Canille R M da S, Belloto M V T, et al. Enteric parasites in HIV-1/AIDS-infected patients from a Northwestern São Paulo reference unit in the highly active antiretroviral therapy era. Rev Soc Bras Med Trop SBMT 2011; 44 (6): 665-9.

10.- Montalvo R, Ticona E, Ñavincopa M, García Y, Chávez G, Chávez V, et al. Diarrea recurrente por Cystoisopora belli en pacientes con infección por VIH con TARGA. Rev Peru Med Exp Salud Publica 2013; 30 (2): 326-30.

11.- Boyles T H, Black J, Meintjes G, Mendelson M. Failure to eradicate Isospora belli diarrhoea despite immune reconstitution in adults with HIV - a case series. PLoS One 2012; 7 (8): e42844.
12.- Lagrange-Xélot M, Porcher R, Sarfati C, de Castro N, Carel O, Magnier J-D, et al. Isosporiasis in patients with HIV infection in the highly active antiretroviral therapy era in France. HIV Med 2008; 9 (2): 126-30.

13.- George M D, Asmuth D M. Mucosal immunity in HIV infection: what can be done to restore gastrointestinal-associated lymphoid tissue function? Curr Opin Infect Dis 2014; 27 (3): 275-81.

14.- El-Taweel H A. Understanding drug resistance in human intestinal protozoa. Parasitol Res 2015; 114 (5): 1647-59.

15.- The INSIGHT START Study Group. Initiation of antiretroviral therapy in early asymptomatic HIV infection. N Engl J Med 2015; 373 (9): 795-807.

16.- DaFonseca S, Niessl J, Pouvreau S, Wacleche V S, Gosselin A, Cleret-Buhot A, et al. Impaired Th17 polarization of phenotypically naive CD4(+) T-cells during chronic HIV-1 infection and potential restoration with early ART. Retrovirology 2015; 38 (12): 1-23.

17.- Verdier R I, Fitzgerald D W, Johnson W D Jr, Pape J W. Trimethoprim-sulfamethoxazole compared with ciprofloxacin for treatment and prophylaxis of Isospora belli and Cyclospora cayetanensis infection in HIV-infected patients. A randomized, controlled trial. Ann Intern Med 2000; 132 (11): $885-8$ 